\title{
Confining vs. conformal scenario for SU(2) with 2 adjoint fermions. Mesonic spectrum.
}

\section{Claudio Pica*}

$C P^{3}$-Origins \& IMADA, University of Southern Denmark, Odense, Denmark

E-mail: pica@cp3.sdu.dk

\section{Luigi Del Debbio}

SUPA, School of Physics an d Astronomy, University of Edinburgh, Scotland, UK

E-mail: luigi.del.debbioded.ac.uk

\section{Biagio Lucini}

School of Physical Sciences, Swansea University, Wales, UK

E-mail: b. lucini@swansea.ac.uk

\section{Agostino Patella}

CERN, Geneva, Switzerland

E-mail: agostino.patella@cern.ch

\section{Antonio Rago}

Department of Physics, Bergische Universität Wuppertal, Germany

E-mail: rago@physik.uni-wuppertal.de

The Minimal Walking Technicolor (MWT) model, based on the SU(2) gauge group with two Dirac adjoint fermions, is expected to lie close to the lower boundary of the conformal window. As such, it is believed to possess a dynamics different enough from QCD to be a viable candidate for a Technicolor theory. We present a detailed analysis of the mesonic spectrum of this model in which we aim to reach the chiral limit while controlling the systematic errors. Comparing our data to the expected behaviors for a QCD-like theory and for an IR conformal theory, we show that the latter scenario is favored.

CP3-Origins-2010-46, CERN-PH-TH/2010-256, WUB/10-31

The XXVIII International Symposium on Lattice Field Theory, Lattice2010

June 14-19, 2010

Villasimius, Italy

\footnotetext{
* Speaker.
} 


\section{Introduction}

The existence of a new strong force in Nature to explain the breaking of Electro-Weak Symmetry dynamically remains one of two the most intriguing possibilities, the other being supersymmetry. Technicolor (TC) models build on our knowledge of the non-perturbative dynamics of QCD. As in the theory of Strong interactions, chiral symmetry breaking occurs in the new postulated sector but at an higher energy scale $\sim \mathrm{TeV}$ leading to Electro-Weak symmetry breaking and providing the correct mass for the Standard Model (SM) gauge bosons. The SM fermions acquire a mass if we further postulate the existence of Extended Technicolor (ETC) interactions.

The first models of TC were based on a scaled up version of QCD, i.e. they were based on a $\mathrm{SU}(\mathrm{N})$ gauge theory with a small number of fundamental matter fields. It was soon realized that such models are not viable candidates. In fact together with the mass of SM fermions, large Flavor Changing Neutral Currents (FCNC) and large values of the Peskin-Takeuchi S parameter would also be generated. Electroweak precision tests performed at LEP tightly constrain the allowed values for FCNC and the oblique parameters, and the scaled up version of QCD is incompatible with the experiments.

The problems of the naive rescaling of QCD could be avoided if the dynamics of the new sector is sufficiently different from QCD. Walking and conformal technicolor theories have been proposed whose large-distance dynamics is expected to be very different from the one of QCD. In particular it was shown that models falling in these frameworks could satisfy the experimental constraints (for recent reviews of techicolor models see [1, 2, 3, 4]).

Good candidate models in these frameworks are those which lie close to the lower boundary of the so-called conformal window, where the presence of an (approximate) IR fixed point is believed to significantly change the non-perturbative dynamics of the theory. The use of matter fields in higher dimensional representations has been recently advocated $[5,6]$ as an effective and economic way of satisfying all known experimental constraints.

Here we will investigate the so-called Minimal Walking Technicolor theory, based on the gauge group SU(2) with two Dirac fermions in the adjoint representation. For a realistic model of Nature the new strong sector of MWT should be complemented by a new $4^{\text {th }}$ family of leptons to cancel the global Witten anomaly and new ETC interactions should be introduced at a higher scale $\Lambda_{\mathrm{ETC}} \gg \Lambda_{\mathrm{TC}} \sim 1 \mathrm{TeV}$ to give mass to the SM fermions.

These unspecified ETC interactions can be taken into account at the TC scale through effective 4-fermion interactions, which we indicate schematically as:

$$
\Delta \mathscr{L} \propto \frac{1}{\Lambda_{E T C}^{2}} \bar{\Psi} \Psi \bar{\Psi} \Psi, \frac{1}{\Lambda_{E T C}^{2}} \bar{\Psi} \Psi \bar{\psi} \psi, \frac{1}{\Lambda_{E T C}^{2}} \bar{\psi} \psi \bar{\psi} \psi
$$

here we indicated with $\Psi$ the new techni-fermions and with $\psi$ the SM fermions fields. From Eq. (1.1) it is clear that a tension exists between obtaining the correct value for the SM fermions, generated by the second kind of interactions, and producing small FCNC, generated by the third kind of interactions. The latter can be suppressed by requiring $\Lambda_{\mathrm{ETC}}$ to be large enough, but then the model should provide an enhancement mechanism for producing a condensate $\langle\bar{\Psi} \Psi\rangle$ much bigger than in QCD. A possible enhancement mechanism is walking. In a walking theory the presence an approximate IR fixed point makes the couplings of the theory evolve very slowly among two 
energy scales, which could ideally be identified with the $\Lambda_{\mathrm{TC}}$ and the $\Lambda_{\mathrm{ETC}}$ scale. If this is the case the also the anomalous dimension of the mass $\gamma(\alpha(\mu))$ will be approximatively constant $\approx \gamma^{*}$ as a function of the sliding energy scale $\mu$, yielding for the condensate:

$$
\langle\bar{\Psi} \Psi\rangle_{E T C} \simeq\langle\bar{\Psi} \Psi\rangle_{T C}\left(\frac{\Lambda_{E T C}}{\Lambda_{T C}}\right)^{\gamma^{*}}
$$

which is, as anticipated, much bigger than in the QCD-like case for which we have $(\gamma(\alpha) \approx k \alpha)$ :

$$
\langle\bar{\Psi} \Psi\rangle_{E T C} \simeq\langle\bar{\Psi} \Psi\rangle_{T C}\left(\ln \frac{\Lambda_{E T C}}{\Lambda_{T C}}\right)^{k}
$$

A few comments are in order. A large value of $\gamma^{*}$ are needed to generate masses to all the SM fermions. When considering the TC strong sector in isolation, larger values of $\gamma^{*}$ are expected near the lower boundary of the conformal window. This value of $\gamma^{*}$ could be modified by the effects of the ETC interactions [7]. Even if the strong TC sector in isolation is conformal, it could still be used as a viable model of TC once small deformations, such as a mass term, are introduced.

The results presented below are obtained for MWT as a strongly coupled theory in isolation and are based on our recent work published in $[8,9,10]$. In this proceeding we will focus our attention only on the mesonic spectrum. A companion contribution [11] presents in details our study of the gluonic sector.

\section{Numerical simulation}

For the numerical simulations of the SU(2) gauge theory with two Wilson-Dirac fermions in the adjoint representation, we used our own HiRep code which we developed from scratch and extensively tested and presented in [12]. All the simulations presented in this work are performed at a fixed lattice spacing, corresponding to a bare coupling $\beta=2.25$. This value of the coupling is chosen to avoid a bulk phase transition present at about $\beta=2.0[13,14,15]$.

The extrapolation towards the continuum limit will require a new series of runs at different values of the bare coupling, and it will be the matter of future investigations.

We show results for four different lattices: $16 \times 8^{3}, 24 \times 12^{3}, 32 \times 16^{3}$ and $64 \times 24^{3}$. We produced for each of these four lattices a number of ensembles corresponding to different quark masses, in the range corresponding to pseudoscalar masses between $0.6 a^{-1}$ and $0.2 a^{-1}$ in units of the lattice spacing $a$.

When using Wilson fermions, the chiral and infinite volume limits are intertwined. In practice for the simulation to be stable, one cannot arbitrarily decrease the quark mass without also increasing the volume. This is also necessary to keep under control finite-volume effects, thus remaining in the large volume phase. To control the size of these systematic errors we perform simulations with different volumes.

For each lattice size and quark mass we produced a statistical ensemble of about 5000 thermalized configurations, except on the largest lattice for which our preliminary result is based on approximately 500 configurations at each quark mass. Further details about the numerical simulations can be found in $[9,10]$. 


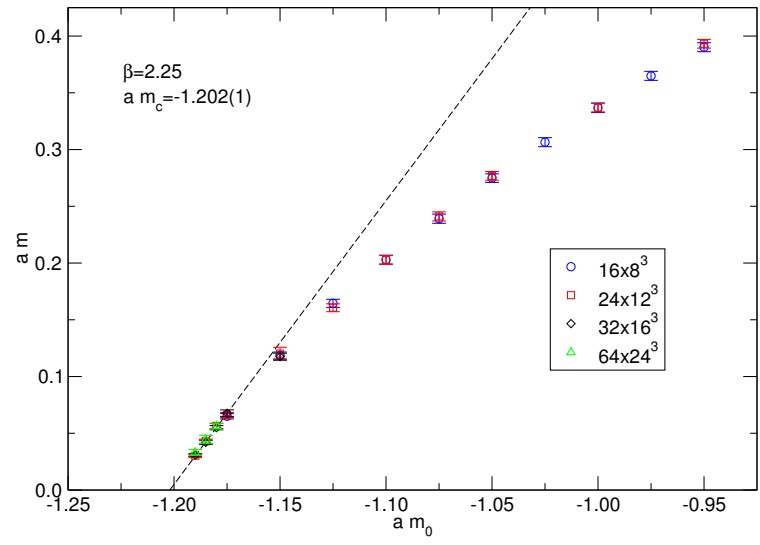

Figure 1: Extrapolation of the quark mass from the axial Ward identity to locate the chiral limit. As expected no significant finite size effects are present.

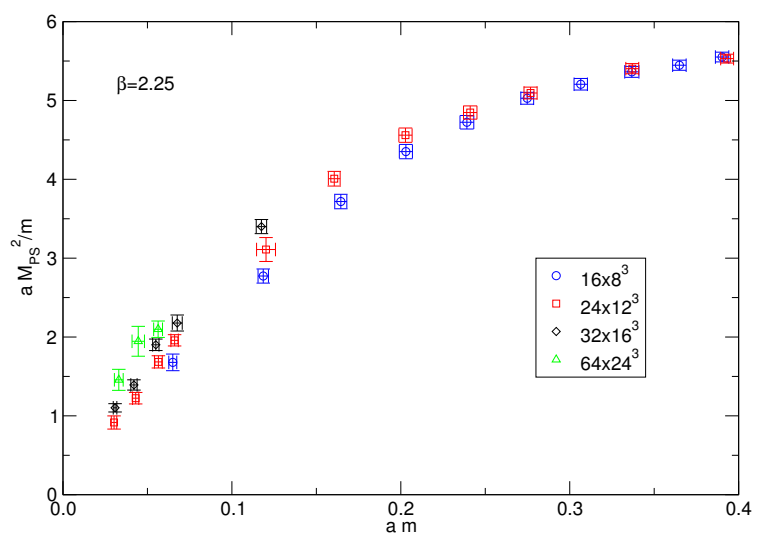

Figure 2: Ratio of the pseudoscalar mass squared to the PCAC mass. The extrapolation to the chiral limit suffers from large finite-volume effects. See the text for a discussion.

For each ensemble of configurations, we measured a set of observables which includes mesonic and gluonic observables. Here we only present result for the mesonic spectrum. The mesonic observables we will consider are the current quark mass from the axial Ward identity (PCAC mass) $m$, the pseudoscalar triplet meson mass $M_{\mathrm{PS}}$, its decay constant $F_{\mathrm{PS}}$, and the vector triplet meson mass $M_{\mathrm{V}}$. Their complete definitions and the numerical methods used to extract these observables on the lattice are described in [9].

\section{Results}

The chiral limit with Wilson fermions is identified with the limit in which the PCAC mass vanishes. The explicit breaking of the chiral symmetry with Wilson fermions induces an additive renormalization of the bare quark mass. In Fig. 1 the measured PCAC mass is shown, as a function of the bare mass $m_{0}$, for different lattice sizes. Being an ultraviolet quantity, this particular observable is insensitive to the physical volume. A linear extrapolation is performed of the four lightest points available, yielding the location of the chiral limit for critical bare mass $a m_{c}=-1.202(1)$.

Our results for the mass of the pseudoscalar meson are presented in Fig. 2. Given the level of accuracy of the measure of the mass, the finite volume effects on $M_{\mathrm{PS}}$ are clearly visible and, as the PCAC mass is decreased, they become more and more pronounced, as expected. To quantify this systematic effect and to keep it under control, we use larger lattices as the chiral limit is approached.

To distinguish between the IR-conformal and the QCD-like case, we plot in Fig. 2 the ratio $a M_{\mathrm{PS}}^{2} / m$. For a QCD-like theory this ratio $a M_{\mathrm{PS}}^{2} / m$ should converge to a non-zero constant in the chiral limit. On the other hand if the theory has an IR fixed point the ratio should vanish in the chiral limit if the anomalous dimension of the mass at the fixed point satisfies $\gamma_{*}<1$ or diverge if $\gamma_{*}>1$. We note that finite volume effects tend to make the ratio $a M_{\mathrm{PS}}^{2} / m$ smaller. However if one assumes a confining scenario and tries a linear extrapolation using the lightest quark masses available in the range am $<0.1$, the extrapolated value remains consistent with zero within $2 \sigma$. We conclude that this is a first hint that our data favor the IR conformal scenario with $\gamma_{*}<1$. An 


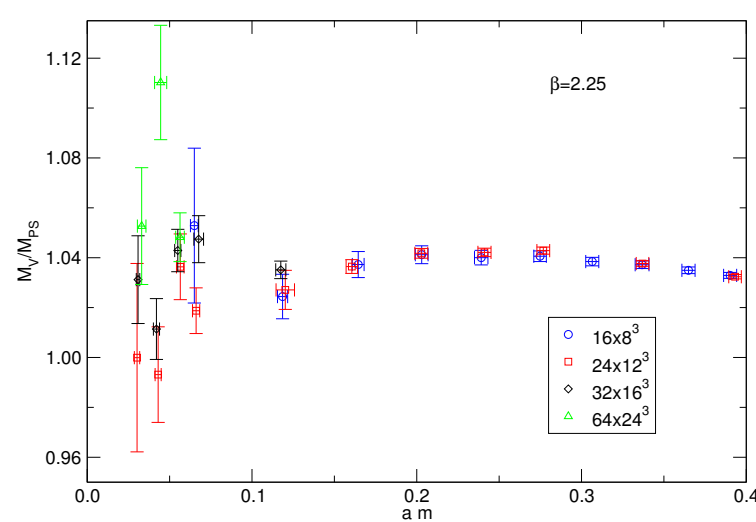

Figure 3: Comparison between the vector and pseudoscalar meson masses. At large PCAC mass, due to quenching the ratio is very near to one. Near the chiral limit large finite size effects show up.

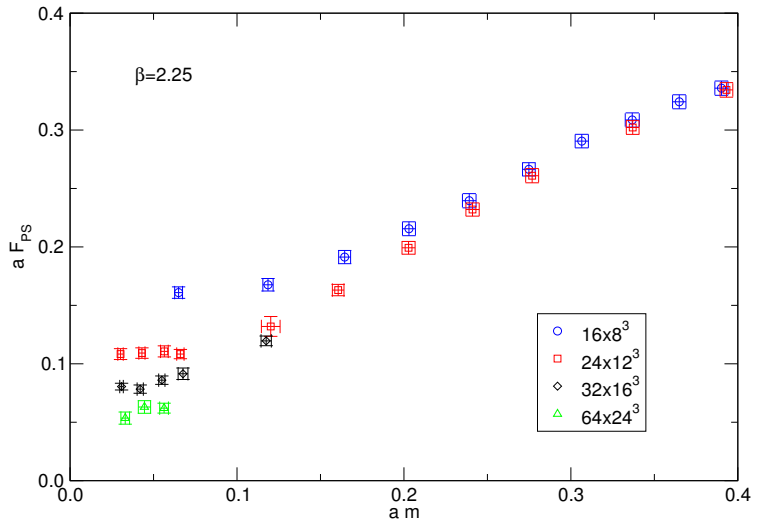

Figure 4: Pseudoscalar decay constant near the chiral limit. Very large finite volume effects are present also in this case which cause the chiral extraplation to be have large uncertainties.

accurate determination of the anomalous dimension is difficult from this measure of $M_{\mathrm{PS}}$ alone, but it is clear from the almost linear behavior of $M_{P S}$ as a function of $m$ that a small value of $\gamma_{*}$ is preferred. The IR conformal scenario is also favored when one looks at the ratio of the vector to the pseudoscalar mesons masses which is shown in Fig. 3. This ratio is bounded to be greater than 1 and it tends to 1 in the heavy quark limit. At large $m$ finite volume effects are small, the ratio is bigger than 1 and decreasing as $m$ increases as expected. What is remarkable in our data is the fact that in the whole mass range we were able to explore, and in which the $M_{\mathrm{PS}}$ changes roughly by a factor of 7 , the vector meson never becomes more than $5 \%$ heavier than the pseudoscalar, so that this ratio remains approximately constant in the chiral limit. This is the expected behavior in an IR conformal theory as in this case all the hadronic masses scale with the same critical exponent in the limit $m \rightarrow 0$.

In Fig. 4 we consider another physically interesting quantity: the pseudoscalar decay constant $F_{\mathrm{PS}}$. This quantity is the one which shows the largest finite-volume effects. The curves defined by $F_{\mathrm{PS}}$ at different volumes, as a function of the PCAC mass tend to a clearly visible envelop for large volumes, which should be used for the chiral extrapolation. For a QCD-like theory the result in the chiral limit is a non-zero value. The direct extrapolation however is difficult to carry out with reasonable accuracy; for this reason, we prefer to exploit the finite-size effects to obtain a more insightful result. It is well known that in the neighborhood of an IR fixed point one can consider the finite size $L$ of the system as a relevant parameter in the RG flux and thus obtain universal scaling laws for physical observables. These finite size scaling can be conveniently rewritten, for the case of the pseudoscalar decay constant, as:

$$
L F_{\mathrm{PS}}=\Upsilon\left(L m^{1 /\left(1+\gamma_{*}\right)}\right) .
$$

Scaling is observed if the different curves obtained by varying the mass and corresponding to different volumes, collapse on top of each other. By varying the critical exponent $\gamma_{*}$ an estimate of its value can be obtained, if scaling is observed. We plot in Fig. 5, the quantity $L F_{\mathrm{PS}}$ as a function of $x=L m^{1 /\left(1+\gamma_{*}\right)}$ for a range of values of $\gamma_{*}$. Good scaling is observed for small values of $\gamma_{*}$, while 


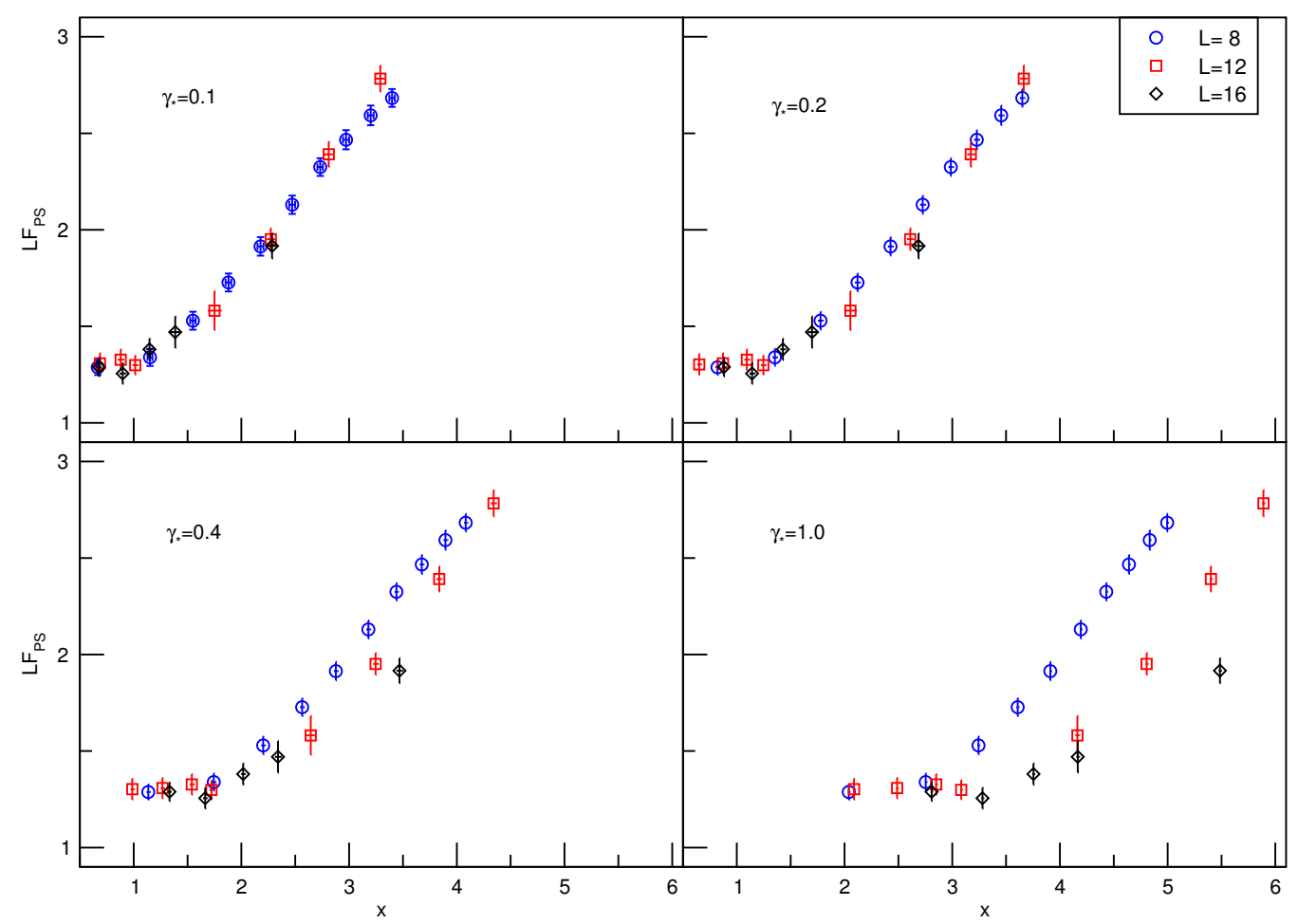

Figure 5: Quality of the scaling of $L F_{P S}$ as a function of $x=L m^{1 /\left(1+\gamma_{*}\right)}$ for various values of $\gamma_{*}$.

large values of $\gamma_{*}$ seem again to be disfavoured. The observed scaling is again in agreement with the existence of an IR fixed point with a small $\gamma_{*}$ in the MWT theory. The range of values of $\gamma_{*}$ for which a good quality of the scaling is obtained is also compatible with independent estimates performed with the Schrödinger functional [16].

\section{Conclusion}

In this work we have presented the results of our measure of the mesonic spectrum of one of the candidate theories for a realistic technicolor model, the so-called Minimal Walking Technicolor, based on gauge group SU(2) with two Dirac adjoint fermions.

Using numerical lattice simulations we found evidence that the MWT theory lies inside the conformal window and possesses an IR conformal fixed point. The evidence provided is based on the behavior of the different mesonic observables, namely the pseudoscalar and vector meson mass and the pseudoscalar decay constant. These quantities show significant deviations from the expectations of a more familiar QCD-like scenario, where spontaneous chiral symmetry breaking occurs. On the other hand our data is compatible with the existence of an IR fixed point when using the scaling laws expected in this case.

Although clear signs of IR conformality were presented, the present study still has several limitations which need to be addressed. The major source of uncertainty is the use of a single lattice spacing in all our numerical simulations. A continuum extrapolation involving different lattice spacings is highly desirable. 
Finally in this proceeding we only discussed the mesonic spectrum, while substantially more information can be obtained by combining it with observables from other sectors of the theory, as we proposed in Ref. [8]. The detailed study of gluonic observables, and their comparison to the mesonic ones is presented in the companion contribution [11], which provides further evidence for the existence of an IR fixed point.

\section{Acknowledgements}

The numerical calculations presented in this work have been performed on the BlueC supercomputer at Swansea university, on a Beowulf cluster partly funded by the Royal Society and on the Horseshoe5 cluster at the supercomputing facility at the University of Southern Denmark (SDU) funded by a grant of the Danish Centre for Scientific Computing for the project "Origin of Mass" 2008/2009. Our work has been partially supported by STFC under contracts PP/E007228/1 and ST/G000506/1. B.L. is supported by the Royal Society (University Research Fellowship scheme), A.P. was supported by the EC (Research Infrastructure Action in FP7, project "HadronPhysics2"). A.R. thanks the Deutsche Forschungsgemeinschaft for financial support.

\section{References}

[1] C. T. Hill and E. H. Simmons, Phys. Rept. 381 (2003) 235 [Erratum-ibid. 390 (2004) 553] [arXiv:hep-ph/0203079].

[2] F. Sannino, arXiv:0804.0182 [hep-ph].

[3] F. Sannino, Acta Phys. Polon. B 40, 3533 (2009) [arXiv:0911.0931 [hep-ph]].

[4] M. Piai, [arXiv:1004.0176 [hep-ph]].

[5] F. Sannino and K. Tuominen, Phys. Rev. D 71, 051901 (2005) [arXiv:hep-ph/0405209].

[6] D. D. Dietrich and F. Sannino, Phys. Rev. D 75, 085018 (2007) [arXiv:hep-ph/0611341].

[7] H. S. Fukano and F. Sannino, Phys. Rev. D 82 (2010) 035021 [arXiv:1005.3340 [hep-ph]].

[8] L. Del Debbio, B. Lucini, A. Patella, C. Pica and A. Rago, Phys. Rev. D 80, 074507 (2009) [arXiv:0907.3896 [hep-lat]].

[9] L. Del Debbio, B. Lucini, A. Patella, C. Pica and A. Rago, Phys. Rev. D 82 (2010) 014509 [arXiv:1004.3197 [hep-lat]].

[10] L. Del Debbio, B. Lucini, A. Patella, C. Pica and A. Rago, Phys. Rev. D 82, 014510 (2010) [arXiv:1004.3206 [hep-lat]].

[11] A. Patella,L. Del Debbio, B. Lucini, C. Pica and A. Rago, PoS(Lattice 2010)068

[12] L. Del Debbio, A. Patella and C. Pica, Phys. Rev. D 81 (2010) 094503 [arXiv:0805.2058 [hep-lat]].

[13] S. Catterall and F. Sannino, Phys. Rev. D 76, 034504 (2007) [arXiv:0705.1664 [hep-lat]].

[14] S. Catterall, J. Giedt, F. Sannino and J. Schneible, JHEP 0811, 009 (2008) [arXiv:0807.0792 [hep-lat]].

[15] A. J. Hietanen, J. Rantaharju, K. Rummukainen and K. Tuominen, JHEP 0905, 025 (2009) [arXiv:0812.1467 [hep-lat]].

[16] F. Bursa, L. Del Debbio, L. Keegan, C. Pica and T. Pickup, Phys. Rev. D 81, 014505 (2010) [arXiv:0910.4535 [hep-ph]]. 